\title{
Study on economic dispatching of wind power consumption considering dynamic game time-of-use price
}

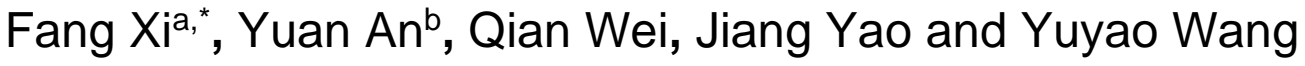 \\ Institute of water Resources and hydro-electric Engineering, Xi'an University of Science and \\ Technology, Xi'an, 710048, China \\ a1354226264@qq.com, b1749847981@qq.com
}

\begin{abstract}
Keywords: Time-of-use price, Dynamic game, Wind power consumption.
Abstract. In the view of the anti-peaking characteristics of wind power, the electric power companies and the users were selected as the object to study and formulate reasonable demand side hourly price mechanism and the demand response model was established based on dynamic game time-of-use price. Proposed model combined with the scheduling optimization of wind power, thermal power and demand response of the three kinds of power generation resources to improve absorptive capacity of wind power and reduce the cost of the system scheduling, and the model mentioned above was solved by an improved particle swarm algorithm in which the simulated annealing theory was introduced. Simulation results showed that compared with the scheduling optimization model under fixed time-of-use electricity price, the model adopted in this paper could effectively reduce the amount of abandoned wind, enhance the running level of the unit and the economy of the system, and prove the effectiveness of the model.
\end{abstract}

\section{Introduction}

Wind power has developed rapidly, which features cleanness, efficiency and sustainable development emission under the context of addressing environmental pollution and energy depletion at present. However, wind power generation is random and fluctuating, and there is a large prediction error [1]. Large scale wind power access increases the difficulty of system operation [2], which, to some extent, inhibits the enthusiasm of the grid to accept wind power [3,4], and it brings challenges to the power grid scheduling operation at the same time. The problem of wind power consumption has become a key factor influencing the development of wind power [5]. Therefore, it is of great concern to wind power development to study how to overcome the randomness of wind power and discuss the large-scale wind power dispatching method.

With the development of the smart grid, the influence of flexible interaction load on the grid operation has gradually been paid more attention [6]. Smart grid uses advanced information technology to dispatch demand-side resources flexibly and realize the interaction of information and electric energy [7], demand response (DR) as an important resource of the interaction framework of smart grid is to guide the users to change the electricity behavior through the price signal or incentive mechanism, which has become one of the important ways of wind power utilization. Thereinto the peak-valley time-of-use price (TOU) can effectively guide users to choose the reasonable power consumption way to actively participate in averting peaks in the current market environment. Reference [8] obtains the conclusion that the model adopted by itself can obviously improve the utilization rate of wind power and reduce the cost of scheduling with considering the elasticity of demand, but in which the model is deterministic without considering the randomness of wind power. [9] aims at the minimum cost of generating coal and starting and ending cost and establishes the optimal dispatching model of wind and fire power under the TOU price by introducing the peak-valley TOU electricity price. This model can reduce the power consumption of coal and increase wind power generating capacity, but without taking into account the interests of the power company. We should consider the relation among government, power companies, users and so on when developing TOU price, and game theory is a good tool to analyze [10]. A model of dynamic game linkage between power generation side and demand side price is given in [11], which can 
objectively allocate the benefit of peak shaving and valley filling brought by TOU price according to the market forces of each link of the system.

Based on the existing research, this paper combines user demand response with game theory to study the electricity pricing mechanism of peak-valley TOU on demand side and establishes economic dispatch model considering time-sharing system with wind power, and analyses and compares the abandoned air volume and the running cost of the system between normal price, fixed price and game price. Examples show that considering the dynamic game TOU price improves the absorptive capacity of wind power and reduces the operation cost of the system.

\section{Study on dynamic game TOU price}

\subsection{Bayes theory}

When we are faced with uncertainty in life, we firstly make a rough guess about the possibility of something happening, and then revise the judgment according to the new information. The prior judgment is called a "priori probability", and the corrected judgment is called the "posterior probability" in statistical language.

Assuming that each participant has I possible types, and $\mathbf{J}$ possible actions, the $\theta^{\mathrm{i}}$ and $\mathrm{a}^{\mathrm{j}}$ are used to represent a given type and a particular action respectively. Supposing that the participant belongs to a priori probability of the type $\theta^{i}$ is $p\left(\theta^{i}\right) \geq 0$, given that the participant belongs $\theta^{i}$, the conditional probability of the participant's choice is $p\left(a^{j} \mid \theta^{i}\right), \sum_{j} p\left(a^{j} \mid \theta^{i}\right)=1$. So,

$$
\operatorname{Pr} o b\left\{a^{j}\right\}=p\left(a^{j} \mid \theta^{1}\right) p\left(\theta^{1}\right)+\cdots+p\left(a^{j} \mid \theta^{I}\right) p\left(\theta^{I}\right)=\sum_{i=1}^{I} p\left(a^{j} \mid \theta^{i}\right) p\left(\theta^{i}\right)
$$

With $\operatorname{Pr} o b\left\{\theta^{i} \mid a^{j}\right\}$ on behalf of the posterior probability, that is, the probability that the participant belongs to the type $\theta^{i}$ in the given case of $a^{j}$. According to the probability formula

$$
\operatorname{Pr} o b\left\{a^{j}, \theta^{i}\right\}=p\left(a^{j} \mid \theta^{i}\right) p\left(\theta^{i}\right)=\operatorname{Pr} o b\left\{\theta^{i} \mid a^{j}\right\} \operatorname{Pr} o b\left\{a^{j}\right\}
$$

The Bayes theory is

$$
\operatorname{Pr} o b\left\{\theta^{i} \mid a^{j}\right\}=\frac{p\left(a^{j} \mid \theta^{i}\right) p\left(\theta^{i}\right)}{\operatorname{Pr} o b\left\{a^{j}\right\}}=\frac{p\left(a^{j} \mid \theta^{i}\right) p\left(\theta^{i}\right)}{\sum_{k=1}^{I} p\left(a^{j} \mid \theta^{k}\right) p\left(\theta^{k}\right)}
$$

\subsection{Formulation of TOU}

According to the cost of TOU price and the historical load, the power supply company sets the TOU price for the purpose of maximizing its own interests, regardless of the cost of transmission and distribution. The objective function is shown in equation (4).

$$
\max F=\sum_{i=1}^{3} P_{f j} Q_{j}+U_{g}
$$

Where, $j=1,2,3$ represents peaks, valleys, and normal periods of the day respectively, $P_{f j}$ is the price of demand side in peak, valley and normal periods, $Q_{j}$ is the load of peak, valley and normal periods after carrying out TOU price, $U_{g}$ indicates the benefits of grid installation from TOU price.

$$
U_{g}=\frac{L_{\max }-L_{\max }^{n}}{1-\tau} J \varphi
$$


Where, $L_{\max }^{n} 、 L_{\max }$ is the maximum load of the nth and initial load curves respectively, MW, $\tau$ is percentage reserve, $J$ is the unit cost of power grid, and $\varphi$ is Periodic annuity coefficient of power grid operation.

Taking the usual period as the average price, the price of the peak period and the valley period can be expressed as

$$
\left\{\begin{array}{l}
P_{f 1}=P_{a v}(1+\varepsilon) \\
P_{f 2}=P_{a v}(1-\delta)
\end{array}\right.
$$

Where, $\varepsilon 、 \delta$ is the proportion of the price rises and decreases on the basis of average electricity price.

Meanwhile, the formulation of TOU price must meet the ceiling price restriction.

$$
P_{f j} \leq P_{f j \max }
$$

Where, $P_{f j \max }$ represents the maximum price limit for the $\mathrm{j}$ period.

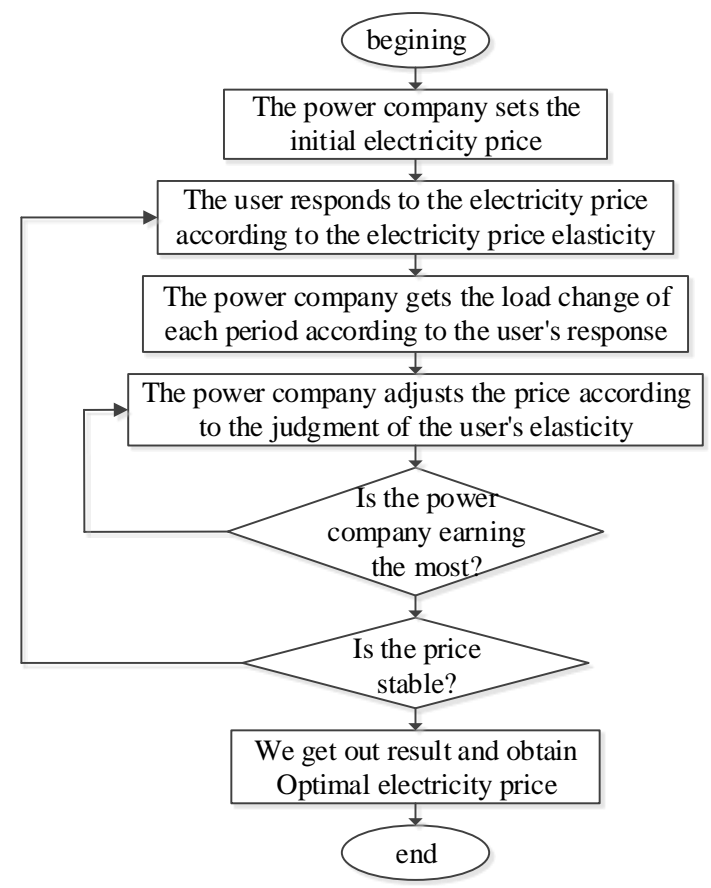

Fig. 1. The flow chart of the game between the power companies and the users

Power companies are faced with users who do not show true elastic information, while power companies need to know that the electricity price elasticity coefficient of the electricity users is high or low, so that different peak and valley price can be formulated according to the elasticity. The way that the power company deals with the incomplete information of users mainly draws on the above Bayes theory, and the concrete implementation process is shown in Fig.1.

\section{Wind power consumption model}

\subsection{Objection function}

In our economic dispatching model including wind power units, the objective function contains two parts: the total electricity purchasing cost of 24 hours a day in the power market by using thermal power unit and wind power unit. Thus the objective function of our economic dispatching model in power system including wind power can be defined as 


$$
\min S F=\sum_{t=1}^{T} \sum_{i=1}^{N}\left[F_{i t}+F_{i t}^{h}+U_{i t}\left(1-U_{i, t-1}\right) F_{q t i}+F_{i t}^{c+}+F_{i t}^{c-}+F_{q t}\right]
$$

In which $F_{i t}, F_{i t}^{h}, F_{q i}$ are the operating costs, environmental costs, start-up/stop-down costs of the thermal power unit $i$ at period $\mathrm{t}$ respectively, and $F_{i t}^{c+} 、 F_{i t}^{c-}$ are the positive and negative rotation reserve capacity costs for thermal power units separately, and $F_{q t}$ is the abandonment cost caused by the abandonment of air during the period $t$.

Where the electricity purchasing cost of thermal power can be expressed as

$$
\begin{aligned}
\sum_{i=1}^{N} F_{h, i t} & =\sum_{i=1}^{N}\left[F_{i t}+F_{i t}^{h}+U_{i t}\left(1-U_{i, t-1}\right) F_{q t i}+F_{i t}^{c+}+F_{i t}^{c-}\right] \\
& =\sum_{i=1}^{N}\left[a_{i} P_{i t}^{2}+b_{i} P_{i t}+c_{i}+\xi\left(\alpha_{i} P_{i t}^{2}+\beta_{i} P_{i t}+\gamma_{i}\right)+U_{i t}\left(1-U_{i, t-1}\right) F_{q t i}+\lambda_{i}^{c+} P_{i t}^{c+}+\lambda_{i}^{c-} P_{i t}^{c-}\right]
\end{aligned}
$$

In which $a_{i}, b_{i}, c_{i}, \xi 、 \alpha_{i}, \beta_{i}$ and $\gamma_{i}$ are the characteristic factors of electricity purchasing cost by using thermal power. And $\lambda_{i}^{c+} 、 \lambda_{i}^{c-}$ are the positive and negative spinning reserve capacity cost coefficient of unit $i$ respectively, Yuan/MW, and $P_{i t}^{c+} 、 P_{i t}^{c-}$ are the positive and negative spinning reserve capacity purchased by the power company partly.

Considering abandoned air cost

$$
F_{q t}=\lambda S P_{t s}
$$

In this equation, $\lambda$ is the cost coefficient of abandoned air, Yuan/MW, $S P_{t s}$ is the abandoned air volume of the wind farm during the period $\mathrm{t}$, MW.

\subsection{Constrains}

Without considering the system loss, the equality constrain is:

$$
\sum_{i=1}^{N} P_{i t}+P_{w t}=D_{t}
$$

And the inequality constrains are:

$$
\begin{gathered}
P_{i \min } \leq P_{i t} \leq P_{i \max } \\
U_{i t} r_{-} d o w n_{i} \leq P_{i t}-P_{i, t-1} \leq U_{i t} r_{-} u p_{i} \\
\left\{\begin{array}{l}
\left(U_{i, t-1}-U_{i t}\right)\left(T_{i, t-1}-T_{i, o n}\right) \geq 0 \\
\left(U_{i t}-U_{i, t-1}\right)\left(-T_{i, t-1}-T_{i, o f f}\right) \geq 0
\end{array}\right. \\
\left\{\begin{array}{l}
0 \leq P_{i t}^{c+} \leq P_{i t}^{e+} \\
0 \leq P_{i t}^{c-} \leq P_{i t}^{e-}
\end{array}\right. \\
P_{w \min } \leq P_{w t} \leq P_{w \max }
\end{gathered}
$$

Where $D_{t}$ is the load value of the system at period $t$ after carrying out TOU price; $P_{i \text { min }} 、 P_{i \max }$ are the lower and upper limit of the ith thermal power unit respectively; and $r_{-} d o w n_{i}, r_{-} u p_{i}$ are the maximum landslide rate and the maximum climbing rate for thermal power unit $i ; P_{w \min } 、 P_{w \max }$ are the minimum and maximum output of wind farm respectively. 


\subsection{Simulation and analysis}

In this paper, the improved particle swarm optimization algorithm quoted simulated annealing idea is utilized to solve the economic dispatching model of wind power consumption taking into account the TOU price. The model has six thermal power units and one wind farm, and the simulations are conducted distinctively on the systems before (case 1) and after the demand response that includes fixed TOU(case 2) and dynamic game TOU(case 3). The basic data of this article are from the literature [12]. According to the model set up in the second part of this paper, the optimal electricity price that can arouse the user's response at different time is shown in table 1, and the load curves in three cases are given in Fig.2.

Table 1. The price in three cases

\begin{tabular}{cccc}
\hline \multirow{2}{*}{ case } & \multicolumn{3}{c}{ Electricity price(Yuan/kWh) } \\
\cline { 2 - 4 } & Peak period & Normal period & Valley period \\
& $10: 00-22: 00$ & $8: 00-10: 00,22: 00-1: 00$ & $1: 00-8: 00$ \\
\hline Case1 & 0.4 & 0.4 & 0.4 \\
\hline Case2 & 0.8 & 0.4 & 0.2 \\
\hline Case3 & 0.785 & 0.4 & 0.168 \\
\hline
\end{tabular}

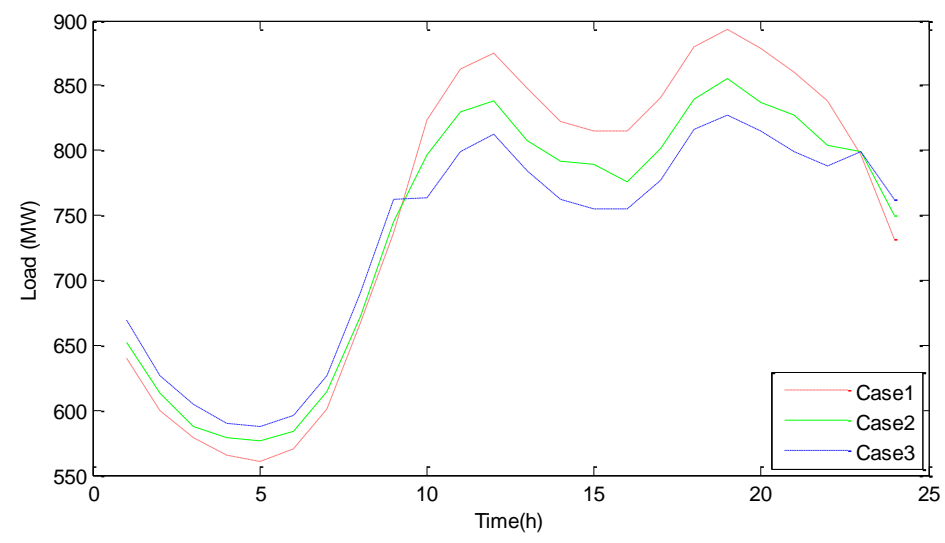

Fig. 2. The load curves in three cases

Fig. 2 shows the load curves of three cases. Due to the existence of TOU price in system, the load curves in three cases are different. According to the trend of curve transformation in 3 cases, we can see that in case 3 , the peak-valley difference of load is $240.5 \mathrm{MW}$, it is more gentle than case 1 and case 2 for shifting load and updating scheduling are gradually to be considered, which improves the positive response of users, relieves the peak energy consumption pressure and plays a certain role in reducing the supply pressure of the power generation side.

The outputs of the thermal power units in three cases are described in Fig.3. Further analysis of the wind power consumption variation before and after the demand response that includes fixed TOU and dynamic game TOU, and the calculation of the amount of abandoned wind power and system cost are shown in Fig. 4. 


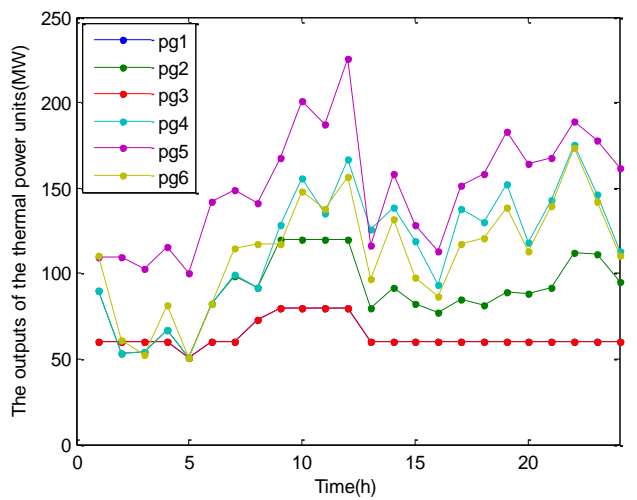

(a).case 1

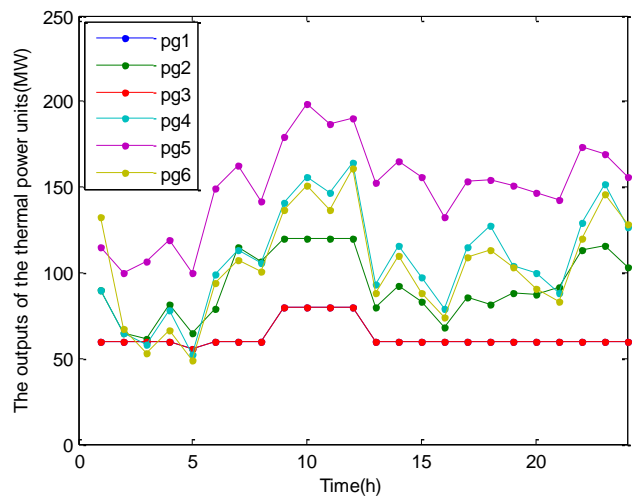

(b).case 2

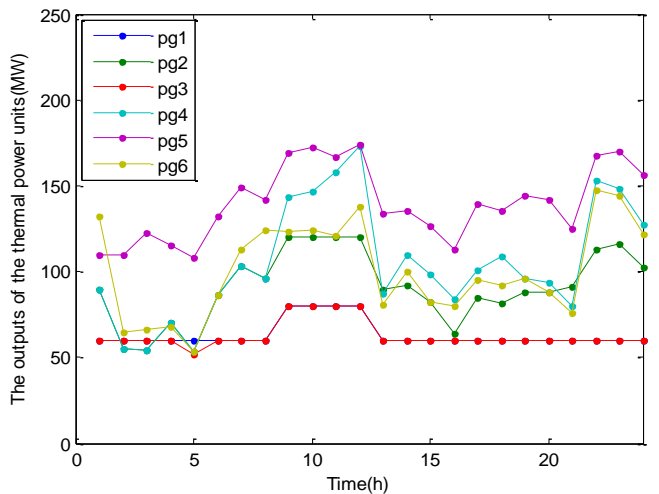

(c).case 3

Fig. 3. The outputs of the thermal power units in various cases

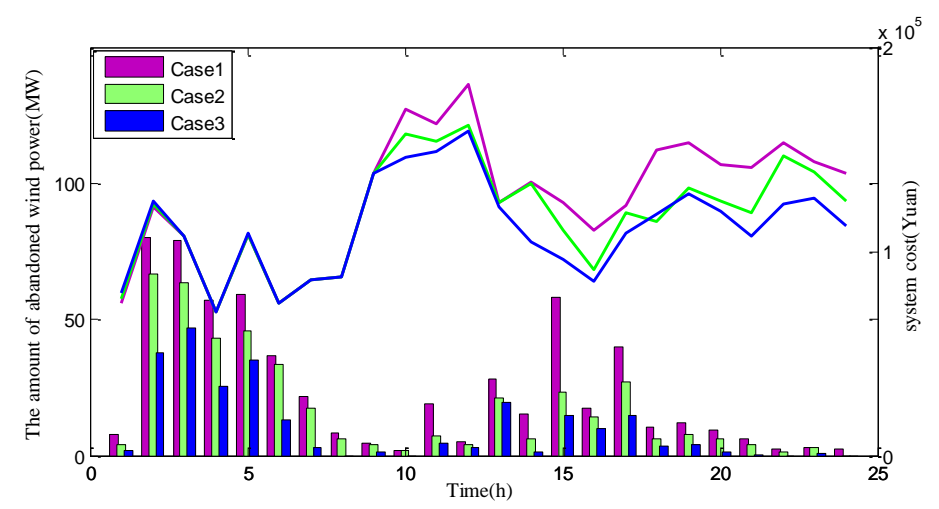

Fig. 4. The amount of abandoned wind power and system cost in three cases

Fig.3 indicates that, in case 3, because of considering dynamic game TOU, the outputs of the thermal power units are obviously reduced and taking the most reduced unit 5 as an example, the fluctuation range of winder power in case 3 is $60 \%$, which is evidently less than case 1 of $126 \%$ and case 2 of $98 \%$.

According to Fig.4, when the dynamic game TOU is taken into consideration, the amount of abandoned wind power has a marked decrease, especially at 3 o'clock in the afternoon, from the original $19.5 \%$ to $5.1 \%$. Meanwhile, the total cost of system reduces from the original 2.8 million Yuan to 2.68 million Yuan. Because the introduction of dynamic game TOU increases the flexibility of the grid, improves the wind power consumption capacity of the grid, so the abandoned wind of cost decreases. And what's more, alongside considering the wind power consumption the total cost of the system reduces to some extent. 


\section{Summary}

In this paper, minimizing the total cost of system based on the wind power consumption as much as possible was taken as the objective function, and the model was optimized with introducing dynamic game TOU price. The simulation results show that compared with fixed TOU, game TOU can effectively reduce the demand for electricity in peak period and transfer part of the load on the peak period to the valley period. Meanwhile, it was demonstrated that the model adopted in this paper can realize the purpose of absorbing the wind power at the lower cost and improve the economy of the system. In addition, this paper introduced an improved particle swarm algorithm based on simulated annealing that can effectively avoid the search being stuck in local minima and improve the convergence speed and precision of the algorithm.

This work provides a theoretical support for analyzing the interaction of participants in the electricity market. Although the game TOU between the power companies and the users is taken into account, the electricity price information on the power generation side should be related to the user side of the price information together to get the most responsive to the market real information of the electricity price mechanism in the mature electricity market.

\section{References}

[1] Chuangying Xiao, Ningbo Wang, and Jing Dou, Power characteristics of Jiuquan wind power base, Int. J. Automation of Electric Power Systems, vol. 34, pp. 64-67, 2010.

[2] Armando M Leite da Silva, Warlley S Sales, and Luiz Antonio da Fonseca Manso, Long-term probabilistic evaluation of operating reserve requirements with renewable sources, Int. J. IEEE Transactions on Power Systems, vol. 25, pp. 106-116, 2010.

[3] Rongfu Sun, Tao Zhang, and Ji Liang, Evaluation and application of wind power integration capacity in power grid, Int. J. Automation of Electric Power Systems, vol. 35, pp. 70-76, 2011.

[4] Feng Li, Yi-chuan Lu, Influences of large-scale wind energy converters on transmission systems, Int. J. Electric Power, vol. 39, pp. 80-85, 2006.

[5] Lingzhi Zhu, Ning Chen, and Hualing Han, Key problems and solutions of wind power accommodation, Int. J. Automation of Electric Power Systems, vol. 35, pp. 29-34, 2011.

[6] Ye Xia, Chongqing Kang, and Bo Ning, A generation and load integrated scheduling on interaction mode on customer side, Int. J. Automation of Electric Power Systems, vol. 36, pp. 17-23, 2012.

[7] BeiBei Wang, Yang Li, Demand side management planning and implementation mechanism for smart grid, Int. J.Electric Power Automation Equipment, vol. 30, pp. 19-24, 2010.

[8] Ram teen Sioshansi Evaluating the impacts of real-time pricing on the cost and value of wind generation. Int. J. IEEE Trans on Power Systems, vol. 25, pp. 741-748, 2010.

[9] Yinhui Zhao, Liwei Ju, and Changqing Xu, Energy-saving generation dispatching optimization model of wind power and thermal power based on time-of-use price, Int. J. Electric Power, vol. 47, pp. 32-36, 2014.

[10] Shaolun Zeng, Yulong Ren, and Jun Li, Game based time-of-use electricity price models and relevant simulations, Int. J. East China Electric Power, vol. 35, pp. 44-48, 2007.

[11]Jun Li, Junyong Liu, and Lianfang Xie, Dynamic game linkge of TOU pricing between generating side retail side, Int. J. Electric Power Automation Equipment, vol. 32, pp. 16-19, 2012. 
[12]Wenying Liu, Jing Wen, and Chang Xie, Multi-objective optimal method considering wind power accommodation based on source-load coordination, Int. J. Proceeding of the CSEE, vol. 35, pp. 1079-1088, 2015. 